\begin{tabular}{|c|c|}
\hline Title & Dynamical analysis on duplicating-and-assimilating process: Toward the understanding of mirror-neuron systems \\
\hline Author(s) & Kang, Hunseok; Tsuda, Ichiro \\
\hline Citation & $\begin{array}{l}\text { Journal of Integrative Neuroscience, 11(4), 363-384 } \\
\text { https://doi.org/10.1142/S0219635212500240 }\end{array}$ \\
\hline Issue Date & $2012-12$ \\
\hline Doc URL & http:/hdl.handle.net/2115/52127 \\
\hline Rights & $\begin{array}{l}\text { Electronic version of an article published as Journal of Integrative Neuroscience, 11, 4, 2012, 363-384, } \\
10.112 / \text {, } 0219635212500240 \Theta \text { copyright World Scientific Publishing Company, } \\
\text { http:/www.worldscientific.com/worldscinet/3in }\end{array}$ \\
\hline Type & article (author version) \\
\hline File Information & JIN11-4_363-384.pdf \\
\hline
\end{tabular}

Instructions for use 


\title{
Dynamical analysis on duplicating-and-assimilating process: toward the understanding of mirror-neuron systems*
}

\author{
Hunseok Kang \\ Department of Mathematics, Soongsil university, 369 Sangdo-ro, Dongjak-gu, \\ Seoul 156-743, KOREA \\ hkang@ssu.ac.kr \\ Ichiro Tsuda ${ }^{\dagger}$ \\ Research Institute for Electronic Science, Hokkaido university, Kita-12, Nishi-6, Kita-ku, \\ Sapporo 060-0812, JAPAN \\ tsuda@math.sci.hokudai.ac.jp
}

Received Day Month Year

Revised Day Month Year

\begin{abstract}
In this paper, we mathematically study a particular process for assimilation in the brain. The research aims to establish a theoretical model at computational level of the mechanism in a cognitive process operated by the mirror-neuron system, to generate a multidimensional system from this model, and to analyze the fundamentals of the related cognitive process in terms of dynamical systems. Finally, to understand the interactions between two individual mirror-neuron systems, we formulate and examine coupled systems that are composed of two distinct systems. We also carry out various numerical simulations to illustrate the assimilation process.
\end{abstract}

Keywords: Mirror-neuron system; Duplicating-and-assimilating process; Embedded bifurcation structure; Coupled systems.

\section{Introduction}

A mirror neuron in monkey and other animals is one of the most remarkable findings in recent brain research [9], [10]. The mirror neurons of one subject respond to voluntary movements of other subjects when such movements are similar to those of the subject, thereby the mirror neurons have been thought to represent sharing of meaning in intentional behaviors. The mirror neurons are now believed to exist even in the human brain in a similar fashion as the so called "mirror-neuron system". In the process of mutual understanding of behaviors in daily-life communications, it

* Supplemental Materials: The readers can find movies on the dynamical trajectories for Figures 3-6 and 8, accessing the following URL: http://www.math.sci.hokudai.ac.jp/ kang/2nd_work_with_IT.html

$\dagger$ Corresponding author. 


\section{H. Kang and I. Tsuda}

would be natural to assume that the dynamic process of assimilation of other movement with one's movement operates. In order to formulate this process, we divide it into three parts: duplicating one's movement, viewing it as the other movement, and assimilating the duplicated one with the original one. Here, we call the dynamic process a duplicating-and-assimilating process, abbreviated as DA-process.

The study in this paper is oriented toward the following three goals:

(1) The first goal is to build a computational model for a cognitive process reflecting upon functional roles in the mirror neurons. We assume in the beginning of the process two distinct states exist; one is the state in which an environmental stimulus is selectively accepted, and the other is the state in which the accepted stimulus is duplicated. The model to be designed should describe how the two distinct states evolve.

(2) The second is to derive a dynamical system from the computational model built above and to analyze it in terms of dynamical systems. We implement various mathematical tools to find dynamical properties which occur during the evolution of the system to an equilibrium, the state of the assimilation process being completed. Then we discuss sufficient conditions to guarantee convergence to the equilibrium.

(3) The third is to investigate coupled systems which are composed of two distinct dynamical systems corresponding to the model. This is for understanding an interactive process between two individual mirror neurons. We link two distinct systems with coupling terms, and then determine whether each system in the coupled system reaches to the assimilated states and whether their assimilated states are the same.

In Sec. 2, we establish a computational model for a cognitive process in the mirror-neuron system based on the functional roles, and formulate a threedimensional dynamical system corresponding to the model. Then, in Sec. 3, we mathematically analyze the dynamical system, find its dynamical properties which lead to an adequate interpretation for the mechanism of the process, and prove some related theoretical results. In Sec. 4, we build some generalized forms of the dynamical system, and examine if they retain the same properties and results. In Sec. 5, we investigate the dynamics of coupled systems in which two individual systems are joined. Finally, Sec. 6 is devoted to summary and discussion.

\section{Mathematical modeling}

Psychologically, a cognition is recognized as a series of mental processes in which an individual accepts the external stimulus, and then, stores and reproduces it. As described in Sec. 1, the mirror-neuron system plays a key role of cognitive process by reproducing others' movements without doing the same movements. In this section, we design a computational model for the DA-process which represents a 


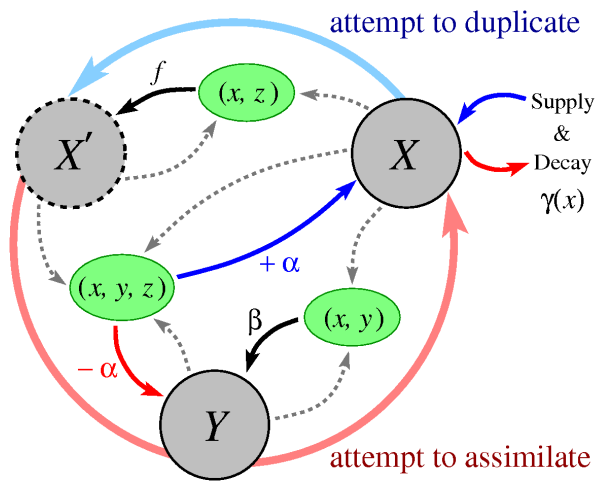

Fig. 1. Duplicating-and-assimilating process in mirror-neuron system.

cognitive process in the mirror-neuron system, and then, derive a dynamical system corresponding to the model.

Figure 1 describes the DA-process conceptually. Let $X$ be an environmental dynamics with consistent external stimuli, $X^{\prime}$ be an internal brain dynamics which is supposed to be operated by the mirror-neuron system, and $Y$ be the dynamics of coupling strength between $X$ and $X^{\prime}$. Also, let $\left\{x_{n}\right\},\left\{y_{n}\right\}$ and $\left\{z_{n}\right\}$ be the sequences of variables which translate the states of the dynamics $X, Y$ and $X^{\prime}$ through the brain, respectively. The variables may represent a population, a volume, or a density of the neurons related to their own functions. From the schematic diagram in Fig. 1, a dynamical system can be derived as follows:

$$
\begin{aligned}
& x_{n+1}=\alpha\left(x_{n}, y_{n}, z_{n}\right)+\gamma\left(x_{n}\right), \\
& y_{n+1}=-\alpha\left(x_{n}, y_{n}, z_{n}\right)+\beta\left(x_{n}, y_{n}\right), \\
& z_{n+1}=f\left(x_{n}, z_{n}\right),
\end{aligned}
$$

where $\alpha, \beta$, and $\gamma$ are functions which are needed to develop the DA process. The function $f$ for $z_{n+1}$ in (2.1) is devised to represent an intermediate state between the dynamics of $X$ and $X^{\prime}$. The computational model described in Fig. 1 formulates the dynamical behaviors through the following three processes:

(1) The dynamics of $X$ describes a situation in which a brain perceives the original movement. The brain receives the information, denoted by $\gamma\left(x_{n}\right)$, obtained from an external supply and a natural decay, and simultaneously yields an operation, denoted by $\alpha\left(x_{n}, y_{n}, z_{n}\right)$, of an interaction among all variables. All of these are used to determine the next state $x_{n+1}$.

(2) The dynamics of $X^{\prime}$ represents the state of duplication via the mirror-neuron system. The mirror-neuron system attempts to selectively duplicate the perceived information. During the duplicating process, a compound of the original state and the duplicated one is produced and used to determine the next state. The state $z_{n+1}$ determined by a function of $x_{n}$ and $z_{n}$, which indicates an intermediate state between $x_{n}$ and $z_{n}$. 
(3) The dynamics of $Y$ is related to the strength of coupling between the states of original stimulus and its duplicated one. The DA process is expected to be slow, so that the variable $y_{n}$ is demanded to be a slow variable. Thus, it is naturally assumed that the coupling strength is negatively proportional to the internal operation $\alpha\left(x_{n}, y_{n}, z_{n}\right)$ like the Le Chatelier-Braun principle, and also, it is dependent on the interaction of $x_{n}$ and $y_{n}$, denoted by $\beta\left(x_{n}, y_{n}\right)$.

We consider the case where the system (2.1) eventually converges to the equilibrium as the three processes described above being repeated. Here, the equilibrium signifies the completion of assimilation. In this case, we look for some the dynamical properties of the system which presents adequate interpretation for the DA process.

In order to organize a specific process of the model from the system (2.1), we place the following assumptions on the dynamics $X, X^{\prime}$ and $Y$ :

(1) The state $x_{n+1}$ is determined by the product of all previous states, that is $x_{n} y_{n} z_{n}$. Also, it decays at a rate which is proportional to the state $x_{n}$, and there exists a constant external supply as well.

(2) The state $z_{n+1}$ is located between the two previous states $x_{n}$ and $z_{n}$, and so, it picks an intermediate value of the two states $x_{n}$ and $z_{n}$.

(3) The state $y_{n+1}$ is accumulative, and it is linearly dependent on the state $x_{n}$, and also, negatively proportional to the product $x_{n} y_{n} z_{n}$.

Based on the assumptions listed above, the functions $\alpha, \beta$ and $\gamma$ are defined as follows:

$$
\alpha\left(x_{n}, y_{n}, z_{n}\right)=b x_{n} y_{n} z_{n}, \quad \beta\left(x_{n}, y_{n}\right)=y_{n}+d x_{n}, \quad \gamma\left(x_{n}\right)=a+c x_{n},
$$

where the parameters $a, b, c$ and $d$ are positive constants. Moreover, the function $f$ takes the following linear convex form for the state $z_{n+1}$

$$
f\left(x_{n}, z_{n}\right)=(1-m) x_{n}+m z_{n}
$$

for some $0<m<1$. Thus, a three-dimensional dynamical system, which describes a specific mechanism in the DA-process, is generated as follows:

$$
\begin{aligned}
& x_{n+1}=c x_{n}+b x_{n} y_{n} z_{n}+a, \\
& y_{n+1}=y_{n}+d x_{n}-b x_{n} y_{n} z_{n}, \\
& z_{n+1}=(1-m) x_{n}+m z_{n} .
\end{aligned}
$$

We investigate the dynamic processes of the system (2.2) in detail. In particular, a model carrying the Hopf bifurcation is a main concern here, because the type of assimilation process may naturally yield oscillatory behaviors. We note that in the case of $x_{n}=z_{n}$ in $(2.2)$, the dynamics $\left\{\left(x_{n}, y_{n}\right)\right\}_{n=0}^{\infty}$ corresponds to the discrete version of Brusselator model. In [5], it was shown that a period-doubling bifurcation and a tangent bifurcation are embedded in the direction field of the Brusselator equation. Being motivated from the study in [5], we provide a detailed examination 
for the existence of the bifurcation structure embedded in a certain orbit of the system (2.2), and use various dynamical systems tools to find mathematical properties of the embedded bifurcation structure which are applicable to the DA process.

\section{Mathematical analysis}

In this section, we analyze the system (2.2) in terms of dynamical systems. We implement various tools in the bifurcation theory to unravel embedded bifurcation structures enclosed in certain orbits of the system, and perform numerical simulations to observe dynamical behaviors of the orbits.

\subsection{System Description}

We define a three-dimensional map $F: \mathbb{R}^{3} \rightarrow \mathbb{R}^{3}, F=\left(f_{1}, f_{2}, f_{3}\right)$, by

$$
\begin{aligned}
& f_{1}(x, y, z)=a+(1-b-d) x+b x y z, \\
& f_{2}(x, y, z)=y+d x-b x y z, \\
& f_{3}(x, y, z)=(1-m) x+m z,
\end{aligned}
$$

where $a, b, d$ and $m$ are positive parameters, and we set $c=1-b-d$ for simplicity of calculation. Then the dynamics of the system (2.2) is given by

$$
\left(x_{n+1}, y_{n+1}, z_{n+1}\right)=F\left(x_{n}, y_{n}, z_{n}\right) .
$$

The map $F$ has a unique fixed point, denoted by $\left(x^{*}, y^{*}, z^{*}\right)$,

$$
\left(x^{*}, y^{*}, z^{*}\right)=\left(\frac{a}{b}, \frac{d}{a}, \frac{a}{b}\right) .
$$

The Jacobian matrix of $F$ at the fixed point $\left(x^{*}, y^{*}, z^{*}\right)$ is

$$
\left.\mathrm{D} F\right|_{\mathbf{x}=\left(x^{*}, y^{*}, z^{*}\right)}=\left[\begin{array}{rrr}
1-b & a^{2} / b & d \\
0 & 1-a^{2} / b & -d \\
1-m & 0 & m
\end{array}\right] .
$$

The characteristic polynomial is

$$
p(\lambda)=(\lambda-(1-b))\left(\lambda-\left(1-\frac{a^{2}}{b}\right)\right)(\lambda-m)-(1-\lambda)(1-m) d,
$$

and from this we obtain the following proposition concerning the stability of the map $F$ at the fixed point $\left(x^{*}, y^{*}, z^{*}\right)$.

Proposition 3.1. If the inequalities

$$
a^{2}<2 b<4, \quad \text { and } \quad-1<m<1,
$$

hold, then there exists a number $d_{0}=d_{0}(a, b, m)>0$ such that whenever $0 \leq d<d_{0}$ holds the fixed point $\left(x^{*}, y^{*}, z^{*}\right)$ of $F$ given in (3.4) is asymptotically stable. 


\subsection{Hopf Bifurcation}

This study aims to mathematically interpret complicated oscillating phenomena in the DA process through the dynamical system (2.2). It can be attained by finding the Hopf bifurcation structure which is smoothly embedded in the system (2.2). We modify the map $F$ to produce a two-dimensional map, and then provide a rigorous mathematical verification of the existence of the bifurcation in the map.

We search for an orbit of $F$ in which the variable $y$ is slowly varied while the others $x$ and $z$ make oscillatory motions. Under a certain condition, indeed, as the system iterates forward, the sequence $\left\{y_{n}\right\}_{n=0}^{\infty}$ in the orbit monotone increases and the difference $\Delta y_{i}=y_{i}-y_{i-1}$ is sufficiently small. (This fact is dealt with in Sec. 3.3.) For this reason, viewing $y$ as a slow variable in the system (3.3) and setting $y=s$ as a parameter, we define a two-dimensional map $F_{s}: \mathbb{R}^{2} \rightarrow \mathbb{R}^{2}$ given by $F_{s}(x, z)=\left(g_{s}(x, z), h_{s}(x, z)\right)$, where

$$
\begin{aligned}
& g_{s}(x, z):=f_{1}(x, s, z)=b s x z+(1-b-d) x+a, \\
& h_{s}(x, z):=f_{3}(x, s, z)=(1-m) x+m z .
\end{aligned}
$$

In this section we assume the following conditions on the parameters:

$$
0<b+d<1 \quad \text { and } \quad 0<m<\frac{1}{2} .
$$

These inequalities are necessary conditions for the existence of the Hopf bifurcation in $F_{s}$.

We first study simple dynamical properties of the map $F_{s}$. It has two fixed points, denoted by $Q_{i}^{*}(s)=\left(q_{i}^{*}(s), q_{i}^{*}(s)\right)(i=1,2)$, where

$$
q_{i}^{*}(s)=\frac{b+d \mp \sqrt{(b+d)^{2}-4 a b s}}{2 b s},
$$

respectively. In order for $q_{i}^{*}(s)$ to be real numbers, we put the following assumption on $s$ in this section:

$$
s \leq s_{(\mathrm{end})}:=\frac{(b+d)^{2}}{4 a b} .
$$

The Jacobian matrix of $F_{s}$ at a point $(x, z)$ is

$$
\left.\mathrm{D} F_{s}\right|_{\mathbf{x}=(x, z)}=\left[\begin{array}{rr}
b s z+1-b-d & b s x \\
1-m & m
\end{array}\right] .
$$

Regarding the parameter $s$ as a bifurcation parameter, we prove the existence of a bifurcation in $F_{s}$. Let $J_{i}(s)(i=1,2)$ be the Jacobian matrices of $F_{s}$ at the fixed points $Q_{i}^{*}(s)$;

$$
J_{i}(s):=\left.\mathrm{D} F_{s}\right|_{\mathbf{x}=Q_{i}^{*}(s)}=\left[\begin{array}{c}
1-\frac{b+d \pm \sqrt{(b+d)^{2}-4 a b s}}{2} \frac{b+d \mp \sqrt{(b+d)^{2}-4 a b s}}{2} \\
1-m
\end{array}\right] .
$$

Its characteristic polynomials $p_{i}^{*}(s)(\lambda)(i=1,2)$ are

$$
p_{i}^{*}(s)(\lambda)=\operatorname{det}\left(J_{i}(s)-\lambda I\right)=\lambda^{2}-\operatorname{tr}\left(J_{i}(s)\right) \lambda+\operatorname{det}\left(J_{i}(s)\right),
$$


where $\operatorname{tr}\left(J_{i}(s)\right)$ and $\operatorname{det}\left(J_{i}(s)\right)$ represent the trace and the determinant of $J_{i}(s)$, respectively. Finally, we denote the discriminants of $p_{i}^{*}(s)(\lambda)$ by $\delta_{i}(s)(i=1,2)$, that are given by

$$
\begin{aligned}
\delta_{i}(s)= & \operatorname{tr}\left(J_{i}(s)\right)^{2}-4 \operatorname{det}\left(J_{i}(s)\right) \\
= & \left(1-m-\frac{b+d \pm \sqrt{(b+d)^{2}-4 a b s}}{2}\right)^{2} \\
& +2(1-m)\left(b+d \mp \sqrt{(b+d)^{2}-4 a b s}\right) .
\end{aligned}
$$

Due to the assumption $m<1 / 2$, the inequality $\delta_{2}(s)>0$ holds so that the eigenvalues of $J_{2}(s)$ are real numbers. Furthermore, at $\lambda= \pm 1$, the characteristic polynomial $p_{2}^{*}(s)(\lambda)$ satisfies

$$
\begin{aligned}
p_{2}^{*}(s)(1) & =-(1-m) \sqrt{(b+d)^{2}-4 a b s}<0, \\
p_{2}^{*}(s)(-1) & =2+2 m+m \sqrt{(b+d)^{2}-4 a b s}-b-d>0,
\end{aligned}
$$

under the assumptions on the parameters given in (3.6). This implies that one of the eigenvalue of $J_{2}(s)$ is always greater than one, and the other is always between -1 and 1 . Thus, the fixed point $Q_{2}^{*}(s)$ is a saddle for all $s<s_{(\mathrm{end})}$, and there is no possibility to have the Hopf bifurcation around $Q_{2}^{*}(s)$. In this respect, it is naturally expected that the bifurcation appears around the other fixed point $Q_{1}^{*}(s)$ if exists. The following claims, $\mathbf{C 1}$ and $\mathbf{C 2}$, can be given as a set of sufficient conditions for the existence of the Hopf bifurcation.

C1. There exists an open interval $\left(s_{1}, s_{2}\right) \subset \mathbb{R}^{-}$such that for each $s \in\left(s_{1}, s_{2}\right)$, the Jacobian matrix of $F_{s}$ at $Q_{1}^{*}(s)$ has complex eigenvalues.

C2. The norm of the complex eigenvalues strictly monotone decreases, and there exists a constant $s_{(\mathrm{crt})} \in\left(s_{1}, s_{2}\right)$ such that the norm of the complex eigenvalues at $s=s_{\text {(crt) }}$ is one. (This means that $s=s_{(\mathrm{crt})}$ is the critical value for the bifurcation point.)

Then there exists the Hopf bifurcation in the system $F_{s}$ owing to the following theorem.

Theorem 3.1. (Generic Hopf bifurcation for discrete dynamical systems; see [3].) For any generic two-dimensional one-parameter family

$$
\mathbf{x} \mapsto f(\mathbf{x}, \alpha),
$$

having at $\alpha=0$ the fixed point $\mathbf{x}=\mathbf{x}^{*}$ with complex multipliers $\mu_{1,2}=e^{ \pm i \theta_{0}}$ there is a neighborhood of $\mathbf{x}^{*}$ in which a unique closed invariant curve bifurcates from $\mathbf{x}^{*}$ as $\alpha$ passes through zero.

We now show that the claim $\mathbf{C 1}$ is always true under the assumptions in (3.6). The discriminant $\delta_{1}(s)$ given in (3.9) and its derivatives $\delta_{1}^{\prime}(s)$ and $\delta_{1}^{\prime \prime}(s)$ are useful 

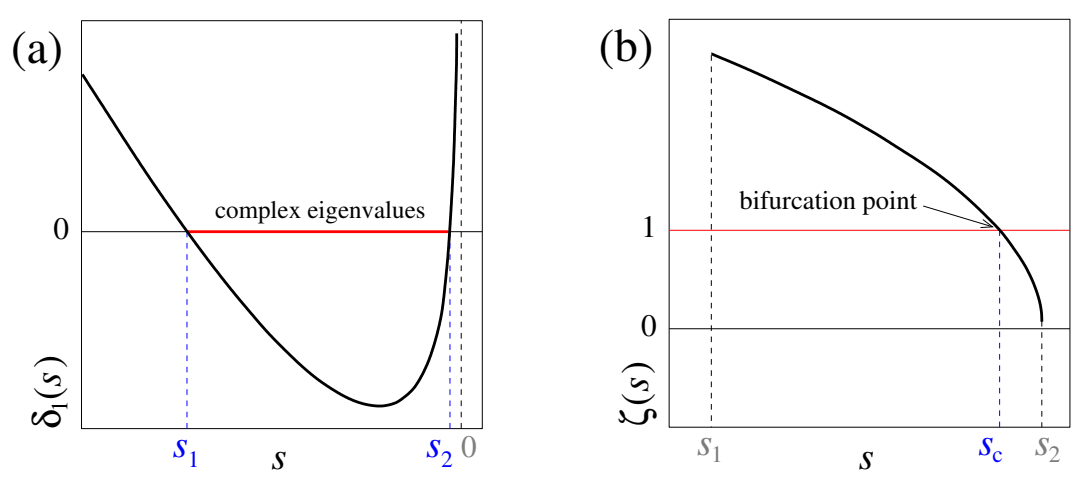

Fig. 2. (a) The discriminant $\delta_{1}(s)$ in (3.9) of the characteristic polynomial of the Jacobian matrix $J_{1}(s)$. For each $s \in\left(s_{1}, s_{2}\right), J_{1}(s)$ has two conjugate complex eigenvalues. (b) The norm $\zeta(s)$ in (3.10) of the complex eigenvalues of $J_{1}(s)$. As $s$ increases from $s_{1}$ to $s_{2}, \zeta(s)$ strictly monotone decreases from $\zeta\left(s_{1}\right)$ to $\zeta\left(s_{2}\right)$, where $\zeta\left(s_{1}\right)>1$ and $0<\zeta\left(s_{2}\right)<1$.

in finding the open interval $\left(s_{1}, s_{2}\right)$ described in $\mathbf{C 1}$. One can easily confirm the following properties:

$$
\lim _{s \rightarrow-\infty} \delta_{1}(s)=+\infty, \quad \text { and }\left.\quad \delta_{1}(s)\right|_{s=0}>0 .
$$

The derivatives $\delta_{1}^{\prime}(s)$ and $\delta_{1}^{\prime \prime}(s)$ are as follows:

$$
\begin{aligned}
& \delta_{1}^{\prime}(s)=\frac{a b(6(1-m)-(b+d))}{\sqrt{(b+d)^{2}-4 a b s}}-a b \\
& \delta_{1}^{\prime \prime}(s)=\frac{2 a^{2} b^{2}(6(1-m)-(b+d))}{\left(\sqrt{(b+d)^{2}-4 a b s}\right)^{3}} .
\end{aligned}
$$

Clearly, the inequality $\delta_{1}^{\prime \prime}(s)>0$ holds for all $s<0$. The equality $\delta_{1}^{\prime}(s)=0$ yields a critical point $s=\hat{s}$,

$$
\hat{s}=-\frac{3(1-m)(3(1-m)-(b+d))}{a b},
$$

and its value $\delta_{1}(\hat{s})$ is

$$
\delta_{1}(\hat{s})=4(1-m)(b+d-1+2 m-1)<0 .
$$

Then due to the assumptions in (3.6), we have

$$
\hat{s}<0, \quad \delta_{1}(\hat{s})<0 \quad \text { and } \quad \delta_{1}^{\prime \prime}(s)>0 .
$$

Thus, there exists an open interval $\left(s_{1}, s_{2}\right)$ with $s_{1}<s_{2}<0$ such that for each $s \in\left(s_{1}, s_{2}\right)$, the Jacobian matrix of $F_{s}$ at $Q_{1}^{*}(s)$ has two complex eigenvalues. See Fig. 2 (a).

On the other hand, we search for a relevant condition of parameters which guarantees that the claim $\mathbf{C 2}$ holds. For each $s \in\left(s_{1}, s_{2}\right)$, the Jacobian matrix $J_{1}(s)$ has 
two complex eigenvalues. We let $\zeta(s)$ be the square of the norm of the eigenvalues. Then $\zeta(s)=\operatorname{det}\left(J_{1}(s)\right)$, and moreover, $\zeta(s)$ is given by

$$
\zeta(s)=m-\frac{b+d}{2}-\left(m-\frac{1}{2}\right) \sqrt{(b+d)^{2}-4 a b s} .
$$

Due to the assumption that $m<1 / 2$ in $(3.6), \zeta(s)$ is always positive, and also, its derivative $\zeta^{\prime}(s)$ is always negative as shown below:

$$
\zeta^{\prime}(s)=\frac{a b(2 m-1)}{\sqrt{(b+d)^{2}-4 a b s}}<0 .
$$

This means that, as $s$ increases, the norm of the complex eigenvalues of $J_{1}(s)$ strictly monotone decreases. For $i=1,2, \delta_{1}\left(s_{i}\right)=0$ and so, the equality in (3.9) yields

$$
\begin{aligned}
\zeta\left(s_{i}\right) & =\operatorname{det}\left(J_{1}\left(s_{i}\right)\right)=\frac{\operatorname{tr}\left(J_{1}\left(s_{i}\right)\right)^{2}}{4} \\
& =(1-2 m \pm \sqrt{1-m} \sqrt{2-2 m-b-d})^{2} .
\end{aligned}
$$

From this equality, one can derive the inequality

$$
m<\frac{b+d-4+\sqrt{(b+d)^{2}-16(b+d)+32}}{4}
$$

which guarantees the following facts:

$$
\zeta\left(s_{1}\right)>1 \text { and } 0<\zeta\left(s_{2}\right)<1
$$

By (3.11) and (3.13), there exists a unique constant $s_{\text {(crt) }} \in\left[s_{1}, s_{2}\right]$ such that $\zeta\left(s_{(\mathrm{crt})}\right)=1$. Consequently, the norm $\zeta\left(s_{1}\right)$ of the complex eigenvalues of $J_{1}(s)$ has the following properties: (1) $\zeta(s)>1$ if $s_{1}<s<s_{(\mathrm{crt})}$; (2) $\zeta(s)=1$ if $s=s_{\text {(crt) }}$; (3) $0<\zeta(s)<1$ if $s_{\text {(crt) }}<s<s_{2}$. See Fig. 2 (b). We summarize these results found in this section in the following theorem.

Theorem 3.2. Suppose that the inequalities $0<b+d<1$ and

$$
0<m<\frac{b+d-4+\sqrt{(b+d)^{2}-16(b+d)+32}}{4}
$$

hold. Then the Hopf bifurcation occurs in the system $F_{s}$ for $s<(b+d)^{2} /(4 a b)$.

Finally, in the following remark, we note a summary about how the stabilities of $Q_{i}^{*}(s)(i=1,2)$ change while $s$ is varied.

Remark 3.1. While the bifurcation parameter $s$ is varied toward $(b+d)^{2} /(4 a b)$ from the below,

(1) the fixed point $Q_{1}^{*}(s)$ changes the stability from repelling to attracting;

(2) the fixed point $Q_{2}^{*}(s)$ remains unstable. It is a saddle point. 


\subsection{Mechanism for embedded bifurcation}

In what follows, we describe the mechanism of how the Hopf bifurcation found in Sec. 3.2 is properly embedded in an orbit of the map $F$. The mechanism of embedding bifurcation is subject to the generic property of the Hopf bifurcation. The generic property means that the basic character of the bifurcation cannot be altered by arbitrarily small perturbations that are smooth in the variables and the bifurcation parameter. See [2] for details of the generic property of the bifurcations.

We suppose that the set of parameters to be used in this section satisfies all the conditions stated in Proposition 3.1 and Theorem 3.2 so that the map $F$ possesses a unique attracting fixed point, and the two-dimensional map $F_{s}$ generates the Hopf bifurcation. As given in Sec. 3.2, let $s_{(\mathrm{crt})}$ be the critical parameter value of the bifurcation point in the map $F_{s}$. By Theorem 3.1, there exists an constant $s_{\text {(init) }}$ such that for all $s \in\left[s_{\text {(init) }}, s_{\text {(end) }}\right]$, there exists a closed invariant curve $\mathcal{C}_{s}$ around the fixed point $Q_{1}^{*}(s)$ of $F_{s}$, that is,

$$
\mathcal{C}_{s}=\left\{(x, s, z) \mid\left(f_{1}(x, s, z), s, f_{3}(x, s, z)\right) \in \mathcal{C}_{s}\right\},
$$

where $f_{1}$ and $f_{3}$ are given in (3.3). Clearly,

(1) for $s_{\text {(init) }} \leq s<s_{\text {(crt) }}, \mathcal{C}_{s}$ is a simple closed curve with a hole;

(2) for $s_{\text {(crt) }} \leq s \leq s_{\text {(end) }}$, it is a single point $\left(q_{1}^{*}(s), s, q_{1}^{*}(s)\right)$.

Given $s_{\text {(init) }} \leq s \leq s_{\text {(end) }}$, each point $(x, s, z) \in \mathcal{C}_{s}$ lies in the neighborhood of $\left(q_{1}^{*}(s), s, q_{1}^{*}(s)\right)$. Then, a small number $\epsilon>0$ can be chosen so that

$$
\left|x-q_{1}^{*}(s)\right|<\epsilon \text { and }\left|z-q_{1}^{*}(s)\right|<\epsilon .
$$

Since $q_{1}^{*}(s)>0$, one can assume that, for all $s \in\left[s_{(\text {init })}, s_{(\text {end })}\right]$, the values of $x$ and $z$ in each point $(x, s, z) \in \mathcal{C}_{s}$ are positive. We build a dynamical orbit $\mathcal{O}_{F}=$ $\left\{\left(x_{n}, y_{n}, z_{n}\right)\right\}_{n=0}^{\infty}$ of $F$ whose initial point $\left(x_{0}, y_{0}, z_{0}\right)$ is such that

$$
y_{0}=s_{(\text {init })} \quad \text { and } \quad\left(x_{0}, z_{0}\right) \in \mathcal{C}_{s_{(\text {init }}} .
$$

Let $\Delta y_{k}$ be the difference of $y_{k}$ from $y_{k+1}$ for each iteration, i.e.,

$$
\Delta y_{k}=y_{k+1}-y_{k}=x_{k}\left(d-b y_{k} z_{k}\right) .
$$

In order for the embedding mechanism to be enabled by the generic property, $\Delta y_{k}$ remains so small that the point $\left(x_{k}, y_{k}, z_{k}\right)$ in $\mathcal{O}_{F}$ is viewed as a small perturbation in variables and parameters in $F_{s}$, and so, it is prevented from being deviated from the bifurcation structure. This requirement is guaranteed by letting $b$ and $d$ be sufficiently small as followed in (3.16).

On the other hand, for all $s$ satisfying $s_{\text {(init) }} \leq s \leq y^{*}$, each point $(x, s, z) \in \mathcal{C}_{s}$ has the following properties:

$$
0 \leq x \leq x^{*} \text { and } 0 \leq z \leq z^{*} .
$$

By applying the inequalities in (3.17) with $x=x_{k}$ and $z=z_{k}$ to the equality in (3.16), one can show that the difference $\Delta y_{k}$ keeps positive so that the orbit $\mathcal{O}_{F}$ moves toward and finally converges to the fixed point $\left(x^{*}, y^{*}, z^{*}\right)$ as $k$ increases. 
This is the reason that, in Sec. 3.2, $y_{k}$ is assumed to be a slow variable in the map $F$. Therefore, under the assumption that $\Delta y_{k}$ is sufficiently small, the orbit $\mathcal{O}_{F}$ can be viewed as a small perturbation of the Hopf bifurcation, and hence, due to the generic property, the Hopf bifurcation structure of $F_{s}$ is properly embedded in the system $(2.2)$.

\subsection{Rate of convergence}

Let $\mathcal{O}_{F}=\left\{\left(x_{n}, y_{n}, z_{n}\right)\right\}_{n=0}^{\infty}$ be the orbit of $F$ whose initial point satisfies the conditions in (3.15). While $\mathcal{O}_{F}$ converges to the fixed point, some parameters in $F$ may be involved in the rate of convergence. As mentioned in Sec. 3.3, the rate of convergence is mainly dependent on how fast the sequence $\left(y_{n}\right)_{n=0}^{\infty}$ in $\mathcal{O}_{F}$ converges to the constant $y^{*}=d / a$. Thus, dominant factors for controlling the rate of convergence are the values of parameters placed in $f_{2}$ in $F$. Due to the stability of $F_{s}$ at $Q_{1}^{*}$, the orbit $X$ appears around the point $\left(q_{1}^{*}(s), s, q_{1}^{*}(s)\right) \in \mathbb{R}^{3}$ for some $s<s_{(\mathrm{end})}$, and then in order to estimate the value of $\Delta y_{k}$ we set $y_{k}=s, x_{k}=q_{1}^{*}(s)+\epsilon_{1}$, and $z_{k}=q_{1}^{*}(s)+\epsilon_{2}$ for sufficiently small $\epsilon_{1}, \epsilon_{2}$. Then we have

$$
\begin{aligned}
\Delta y_{k} & =x_{k}\left(d-b y_{k} z_{k}\right)=\left(q_{1}^{*}(s)+\epsilon_{1}\right)\left(d-b s\left(q_{1}^{*}(s)+\epsilon_{2}\right)\right) \\
& =a-b q_{1}^{*}(s)+o\left(s, \epsilon_{1}, \epsilon_{2}\right),
\end{aligned}
$$

where the function $o$ in (3.18) depends on $\epsilon_{1}, \epsilon_{2}$ and $s$, and it vanishes as $\epsilon_{1}$ and $\epsilon_{2}$ are sufficiently small. Through the estimation of $\Delta y_{k}$ in (3.18), it is possible to describe how each parameter has an effect on the rate of convergence as follows:

(1) The parameter $a$ is a decisive factor that governs the rate of convergence. As seen in the estimation in (3.18), $\Delta y_{k}$ is proportional to the value of $a$. This means that as $a$ increases the movement of $\mathcal{O}_{F}$ toward the fixed point becomes faster.

(2) The parameter $b$ is a factor, but is not critical. The difference $\Delta y_{k}$ is linearly proportional to the value of $-q_{1}^{*}(s)$. However, since $q_{1}^{*}(s)>0$ is relatively small, $\left|\Delta y_{k}\right|$ rarely varies, no matter whether $b$ increases or decreases.

(3) The parameter $d$ is a factor, but is not critical. A simple calculation shows that $d$ is inversely proportional to the value of $q_{1}^{*}(s)$.

(4) The parameter $m$ does not appear in the estimation of $\Delta y_{k}$ in (3.18). This implies that $m$ is not a factor which change the rate of convergence.

The parameter $m$ has nothing to do with the rate of convergence of the orbit $\mathcal{O}_{F}$, and instead, as seen in Proposition 3.1 and Theorem 3.2, it plays a critical role in determining whether the orbit $\mathcal{O}_{F}$ converges to the fixed point and whether it possesses the embedded bifurcation structure.

The dynamics $X^{\prime}$ is supposed to describe the operation of the mirror-neuron system, and the state of $X^{\prime}$ is determined by the function $f_{3}$ which is entirely dependent on the parameter $m$. This means that the characteristics of the mirrorneuron system of an individual determine whether it reaches to the assimilation 

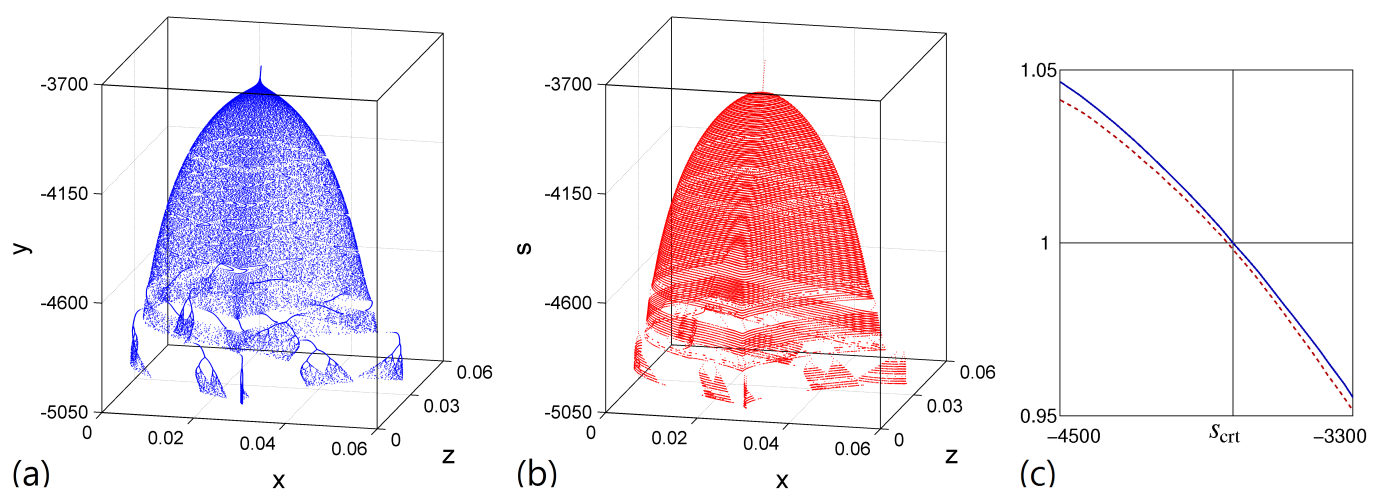

Fig. 3. Comparison of a dynamical orbit with a bifurcation diagram. The values of the parameters in the system are $a=0.03, b=0.01, d=0.02$, and $m=0.05$. (a) A part of the orbit of $F$ originating in $(0,-5000,0)$. The entire orbit eventually converges to the fixed point $(3,2 / 3,3)$. (b) A part of the bifurcation diagram of $F_{s}$. The bifurcation parameter $s$ is varied from $s=-5000$ to $s=-3700$ with the increment $\Delta s=1$. (c) The norm(blue solid line) and the imaginary part(red dotted line) of the complex eigenvalues of $J_{1}(s)$. As $s$ increases, those values monotone decrease. This shows that $s_{\text {crt }} \approx-3831.45$ is the critical parameter value of $s$ for the bifurcation point.

state. Once the conditions of the assimilation state are guaranteed through $f_{3}$, other properties of $\mathcal{O}_{F}$ such as the rate of convergence mainly depend on $a, b$ and $d$.

\subsection{Numerical example}

We finally provide a relevant example of an embedded bifurcation structure in the system (2.2), and compare it to the corresponding Hopf bifurcation of the map $F_{s}$. The parameters are given as follows: $a=0.03, b=0.01, d=0.02$, and $m=0.05$. Then the map $F=\left(f_{1}, f_{2}, f_{3}\right)$ is given by

$$
\begin{aligned}
& f_{1}(x, y, z)=0.03+0.97 x+0.01 x y z \\
& f_{2}(x, y, z)=y+0.02 x-0.01 x y z \\
& f_{3}(x, y, z)=0.95 x+0.05 z
\end{aligned}
$$

The map $F$ has a unique fixed point, $\left(x^{*}, y^{*}, z^{*}\right)=(3,2 / 3,3)$. The eigenvalues of the Jacobian matrix of $F$ at the fixed point are

$$
\lambda_{1} \approx 0.0282, \quad \lambda_{2} \approx 0.9864, \quad \text { and } \quad \lambda_{3} \approx 0.9354,
$$

and so, its fixed point is attracting.

On the other hand, the map $F_{s}=\left(g_{s}, h_{s}\right)$ is given by

$$
\begin{aligned}
& g_{s}(x, z)=0.01 s x z+0.97 x+0.03, \\
& h_{s}(x, z)=0.95 x+0.05 z .
\end{aligned}
$$

One can easily show that the inequality $\delta_{1}(s)<0$ holds, where $\delta_{1}(s)$ is the norm of complex eigenvalues which is defined in (3.9), one can determine the values of $s_{i}$ $(i=1,2)$

$$
s_{1} \approx-100858.55 \text { and } s_{2} \approx-94.78
$$


This implies that for all $s \in\left(s_{1}, s_{2}\right)$ the Jacobian matrix of $F_{s}$ at the fixed point $Q_{1}^{*}(s)$ has complex eigenvalues, and moreover, the critical value of the bifurcation parameter $s=s_{(\mathrm{crt})}$ for the Hopf bifurcation point is obtained from the equality $\zeta(s)=1$, that is, $s_{(\mathrm{crt})} \approx-3831.45$. By numerical tests, one can find a proper value for $s_{\text {(init) }}$ between $s_{1}$ and $s_{\text {(crt) }}$ at which the bifurcation begins. Here, $s_{\text {(init) }}=-5000$ is selected. Figure 3 (b) displays the Hopf bifurcation in the map $F_{s}$. For each $s \in\left[s_{\text {(init) }}, s_{\text {(crt) }}\right]$, the orbit around the bifurcation point is in the shape of a limit cycle. This is because the orbits follow the iteration of the irrational rotational map. Such a behavior of the orbits is guaranteed by the Jacobi's theorem (see [3]), which says that any orbit on the unit circle $S_{1}$ by an irrational rotational map is dense in $S_{1}$.

On the other hand, we consider an orbit $\mathcal{O}_{F}=\left\{\left(x_{n}, y_{n}, z_{n}\right)\right\}_{n=0}^{\infty}$ of $F$ whose initial point $\left(x_{0}, y_{0}, z_{0}\right)$ is $(0,-5000,0)$. As observed in Fig. 3 , for each $s \in\left[s_{\text {(init) }}, s_{\text {(crt) }}\right]$, the orbit $\mathcal{O}_{F}$ moves along the curve $\mathcal{C}_{s}$ and the sequence $\left\{y_{k}\right\}_{k=0}^{\infty}$ in $\mathcal{O}_{F}$ gradually increases. For each $s \in\left[s_{(\mathrm{crt})}, s_{(\mathrm{end})}\right]$, the orbit $\mathcal{O}_{F}$ appears, and finally converges to the fixed point. In this manner, the Hopf bifurcation structure is embedded in $X$. See Fig. 3 (a) for the orbit.

\section{Generalizations}

The state of the dynamics of $X^{\prime}$ is determined by the mirror neuron, and it depends on the characteristic of each individual. In the map $F(3.3)$, a linear form $f_{3}$ is used to express the state of $X^{\prime}$, but there is a limitation in representing the diversity operations of various kinds of mirror neurons. In this respect, we introduce some generalized forms of the map $F$ in (3.3) by replacing the linear polynomials of the map $f_{3}$ by nonlinear polynomials or products of linear polynomials. Two cases are considered, and in each case, one can verify the existence of the embedded bifurcation similarly to Sec. 3.2 and the detailed justification is omitted.

\subsection{CASE I}

We replace the function $f_{3}$ in the map $F$ in (3.3) by the following nonlinear form

$$
f_{3}(x, y, z)=(1-m) x+\frac{m b^{k-1}}{a^{k-1}} z^{k}, \quad \text { for } k \in \mathbb{N},
$$

while keeping the other functions $f_{1}$ and $f_{2}$ in the same form, to produce an extended map, denoted by $F_{1}$. In particular, when $k=1$ in (4.20), the map $F_{1}$ has exactly the same form of $F$. When $k \geq 2, F_{1}$ has more than two fixed points, and one of them is $(a / b, d / a, a / b)$, which is the same as the unique fixed point of $F$. In order to keep the stability of the fixed point $(a / b, d / a, a / b)$ as an attracting one, the assumption on $m$ in (3.5) is replaced by $-1 / k<m<1 / k$, while the other conditions being kept. We deal with an example of a nonlinear case of $f_{3}$ at $k=2$. We set the parameter values as follows: $a=0.02, b=0.05, d=0.02$, and $m=0.2$. Then 
(a)

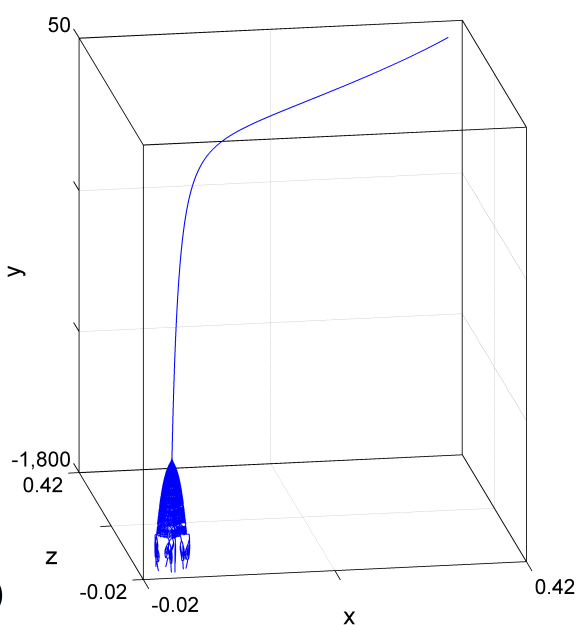

(b)

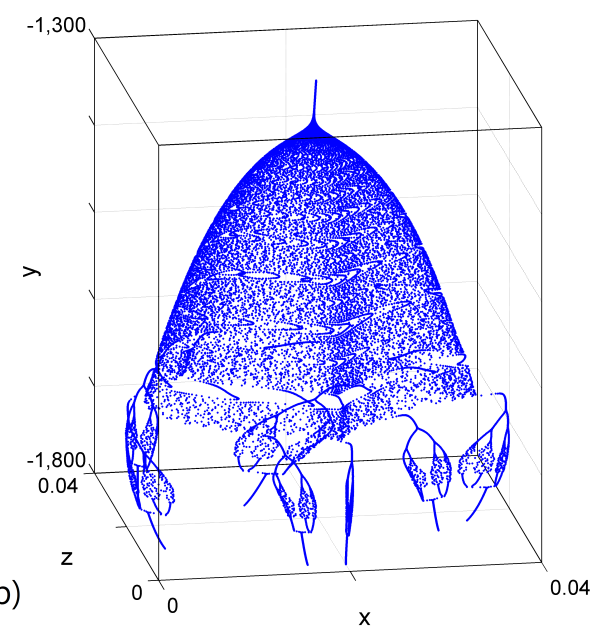

Fig. 4. CASE I in Sec. 4.1. (a) The entire orbit from $(0,-1800,0)$. It converges to a fixed point $(2 / 5,1,2 / 5)$. (b) A part of the orbit that lies between -1800 and -1300 .

(1) one of the fixed points of $F_{1}$ is $(2 / 5,1,2 / 5)$;

(2) it is an attracting fixed point.

Thus, one can build a dynamical orbit which originates in $(0,-1800,0)$ and converges to the fixed point $(2 / 5,1,2 / 5)$, as seen in Fig. 4.

\subsection{CASE II}

We apply another generalized form of $f_{3}$ as follows:

$$
f_{3}(x, y, z)=\left(\left(\sqrt[k]{\frac{b^{k-1}}{a^{k-1}}}-m\right) x+m z\right)^{k},
$$

where $k \in \mathbb{N}$. Let us denote this system by $F_{2}$. Similarly to $C A S E I$, when $k=1$, $f_{3}$ in (2.2) is the linear form in (4.21), and also, the point $(a / b, d / a, a / b)$ is one of the fixed points of $F_{2}$. Let $k=2$ and set the parameter values as follows: $a=0.05$, $b=0.06, d=0.02$, and $m=0.1$. Then we have

(1) one of the fixed points is $(5 / 6,2 / 5,5 / 6)$;

(2) the norms of the eigenvalues of the Jacobian matrix at $(5 / 6,2 / 5,5 / 6)$ are less than one.

Then one can build a dynamical orbit which is originating in $(0,-3650,0)$ and converging to the fixed point $(5 / 6,2 / 5,5 / 6)$. See Fig. 5 .

\section{Coupled systems}

We have studied the DA process which is independently operated by a single mirrorneuron system. However, it is more natural in real life that mirror-neuron system 
(a)

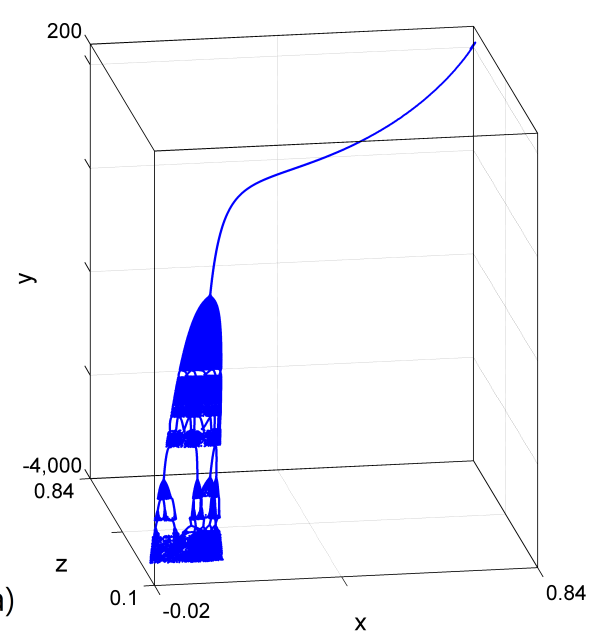

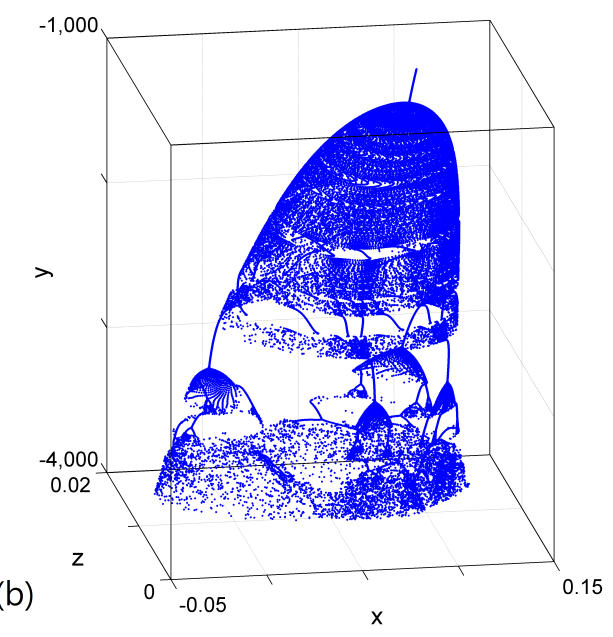

Fig. 5. CASE II in Sec. 4.2. (a) The entire orbit from $(0,-3650,0)$. It converges to a fixed point $(5 / 6,2 / 5,5 / 6)$. (b) A part of the orbit that lies between -3650 and -900 .

of each individual interacts indirectly with others' system to perform their own DA process. For this reason, we consider the case where two distinct systems are linked with various coupling terms and have the same or different initial conditions. There may be various types of two-coupled systems, and among these we deal with three cases of two-coupled systems. In each case, we determine whether two distinct systems in the coupled system converge to their equilibria, and examine whether the equilibriums are the same.

The two-coupled system to be considered is built in the following way. We define a two-coupled system $\left\{\left(\mathbf{x}_{k}, \hat{\mathbf{x}}_{k}\right) \in \mathbb{R}^{3} \times \mathbb{R}^{3} \mid k=0,1,2, \ldots\right\}$ by

$$
\left(\mathbf{x}_{n+1}, \hat{\mathbf{x}}_{n+1}\right)=\left(G\left(\mathbf{x}_{n}, \hat{\mathbf{x}}_{n}\right), \tilde{G}\left(\mathbf{x}_{n}, \hat{\mathbf{x}}_{n}\right)\right),
$$

where, for $\mathbf{x}, \hat{\mathbf{x}} \in \mathbb{R}^{3}$,

$$
\begin{aligned}
& G(\mathbf{x}, \hat{\mathbf{x}})=\left(f_{1}(\mathbf{x}), f_{2}(\mathbf{x}), f_{3}(\mathbf{x})\right)+\left(\rho_{1}(\mathbf{x}, \hat{\mathbf{x}}), 0,0\right) \\
& \tilde{G}(\mathbf{x}, \hat{\mathbf{x}})=\left(\tilde{f}_{1}(\hat{\mathbf{x}}), \tilde{f}_{2}(\hat{\mathbf{x}}), \tilde{f}_{3}(\hat{\mathbf{x}})\right)+\left(\rho_{2}(\mathbf{x}, \hat{\mathbf{x}}), 0,0\right),
\end{aligned}
$$

and $\rho_{i}: \mathbb{R}^{3} \times \mathbb{R}^{3} \rightarrow \mathbb{R}(i=1,2)$ are real valued functions which are involved in the coupling strength between the two systems $\left\{\mathbf{x}_{n}\right\}$ and $\left\{\hat{\mathbf{x}}_{n}\right\}$. Here, the maps $f_{1}, f_{2}$, $\tilde{f}_{1}$ and $\tilde{f}_{2}$ take the forms given in (3.3), and the maps $f_{3}$ and $\tilde{f}_{3}$ may take the linear form given in (3.3) or the generalized one given in (4.20) or (4.21).

\subsection{CASE I}

In this case, a two-coupled system is composed of two systems which are both governed by the map $F$ in (3.3) with the same parameters. We assume that the two systems originate in different initial points. More specifically, the coupled system 


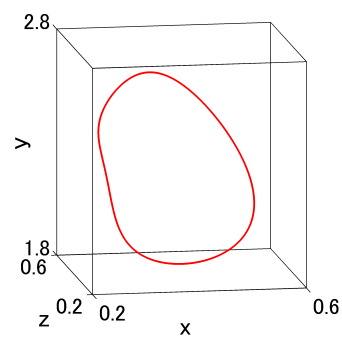

(a) $\gamma=0.01$

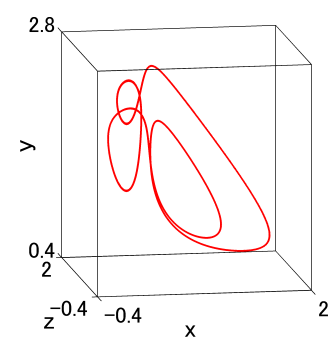

(e) $\gamma=0.028$

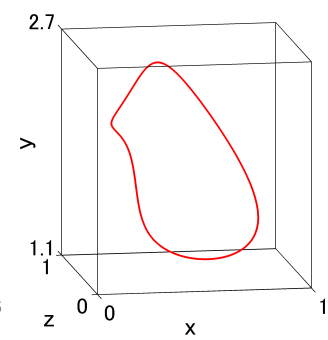

(b) $\gamma=0.016$

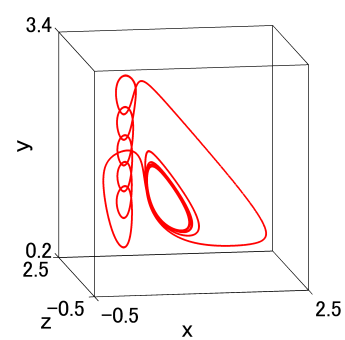

(f) $\gamma=0.029$

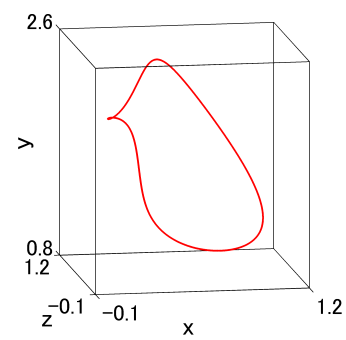

(c) $\gamma=0.02$

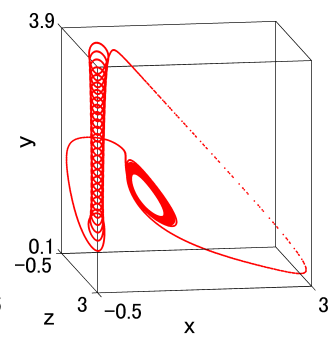

(g) $\gamma=0.0297$

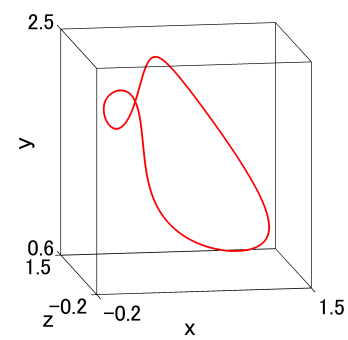

(d) $\gamma=0.025$

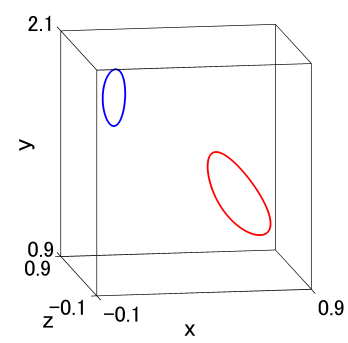

(h) $\gamma=0.03$

Fig. 6. CASE I in Sec. 5.1. The limiting behaviors of the orbits in the systems $\mathbf{A}_{1}$ and $\mathbf{B}_{1}$ according to the coupling strength $\gamma$. For (a)-(g), one of the orbits is drawn, because the other has the same behavior. For (h), both orbits are drawn, because each orbit rotates along its own distinct closed curve. For the numerical simulation, we set $\epsilon=0.005$, and the values $r_{i}(i=1, \ldots, 6)$ are $r_{1}=0.8147$, $r_{2}=0.9058, r_{3}=0.1270, r_{4}=0.9134, r_{5}=0.6324$ and $r_{6}=0.0975$.

is determined by two systems $\mathbf{A}_{1}=\left\{\left(x_{k}, y_{k}, z_{k}\right)\right\}_{k=0}^{\infty}$ and $\mathbf{B}_{1}=\left\{\left(u_{k}, v_{k}, w_{k}\right)\right\}_{k=0}^{\infty}$, which are defined by

$$
\begin{array}{ll}
\mathbf{A}_{1}: & \left(x_{n+1}, y_{n+1}, z_{n+1}\right)=F\left(x_{n}, y_{n}, z_{n}\right)+\left(\gamma\left(x_{n}-u_{n}\right), 0,0\right) \\
\mathbf{B}_{1}: & \left(u_{n+1}, v_{n+1}, w_{n+1}\right)=F\left(u_{n}, v_{n}, w_{n}\right)+\left(\gamma\left(u_{n}-x_{n}\right), 0,0\right),
\end{array}
$$

where the map $F$ is given in (3.3), and $\gamma \in \mathbb{R}$ is a constant which plays the role of a coupling strength between $\mathbf{A}_{1}$ and $\mathbf{B}_{1}$.

In order to have an example, we set the parameters $a=0.02, b=0.06, d=$ 0.05 , and $m=0.05$. Clearly, both systems $\mathbf{A}_{1}$ and $\mathbf{B}_{1}$ have the same fixed point $(1 / 3,5 / 2,1 / 3)$ when they are uncoupled, i.e., when the coupling constant $\gamma$ vanishes. The initial conditions are randomly chosen as follows:

$$
\begin{aligned}
\left(x_{0}, y_{0}, z_{0}\right) & =\left(\epsilon r_{1}, s_{\text {(init) }}+\epsilon r_{2}, \epsilon r_{3}\right), \\
\left(u_{0}, v_{0}, w_{0}\right) & =\left(\epsilon r_{4}, s_{\text {(init) }}+\epsilon r_{5}, \epsilon r_{6}\right),
\end{aligned}
$$

where $s_{\text {(init) }}=-1100, r_{i}(i=1, \ldots, 6)$ are random numbers with $0<r_{i}<1$, and $\epsilon>0$ is a small number that controls the perturbations of the initial conditions.

Under these choices of parameters and initial conditions, we carry out numerical computations to observe the dynamical behavior of the coupled system, and especially, we decide whether two systems $\mathbf{A}_{1}$ and $\mathbf{B}_{1}$ are bounded and convergent and look into their asymptotic behaviors. From numerical tests, it is observed that when 
$\gamma \geq 0.13$, the orbits of $\mathbf{A}_{1}$ and $\mathbf{B}_{1}$ escape to infinity but when $\gamma \leq 0.12$, both systems $\mathbf{A}_{1}$ and $\mathbf{B}_{1}$ are bounded and exhibit the bifurcation structure at the beginning, and follow the patterns which are described in Sec. 3.3 as approaching the fixed point. While $\gamma$ increases from 0 to 0.12 , a variety of dynamical phenomena occur around the fixed point. These phenomena can be naturally interpreted as plausible results which are observable in the interaction of the mirror neurons. The observation and its corresponding interpretation are as follows.

(1) When $0 \leq \gamma \leq 0.008$, the orbits of $\mathbf{A}_{1}$ and $\mathbf{B}_{1}$ eventually converge to the fixed point $(1 / 3,5 / 2,1 / 3)$. Both individual systems in the coupled system eventually reach to the same assimilated state.

(2) When $0.009 \leq \gamma<0.03$, the orbits does not converge to the fixed point, and instead, they rotate around the fixed point along the same simple closed curve without meeting at the same point. (See Fig. 6 (a)-(g).) Both systems fails to converge to a static assimilated state, but they interact each other and repeat the same movement with a constant time difference.

(3) When $\gamma=0.03$, the orbits are bounded and do not converge to the fixed point. Unlike the previous observation, each orbit rotates along a distinct simple closed curve. (See Fig. 6 (h).) Each system makes its own repeated movement which is distinct from the other, and thus they are not assimilated.

(4) When $0.03<\gamma<0.12$, both orbits begin with bifurcation structure and approaches the fixed point, but they eventually escape to infinity. This implies that the systems are not assimilated.

\subsection{CASE II}

We consider another coupled system that is composed of two different forms of systems, denoted by $\mathbf{A}_{2}$ and $\mathbf{B}_{2}$. We assume that the two systems have the same values of parameters $a, b, d$ and $m$, and their initial points are the same. The system $\mathbf{A}_{2}=\left\{\left(x_{k}, y_{k}, z_{k}\right)\right\}_{k=0}^{\infty}$ and $\mathbf{B}_{2}=\left\{\left(u_{k}, v_{k}, w_{k}\right)\right\}_{k=0}^{\infty}$ follow the dynamics which are given by

$$
\begin{aligned}
x_{n+1} & =a+(1-b-d) x_{n}+b x_{n} y_{n} z_{n}+\gamma\left(x_{n}-u_{n}\right), \\
y_{n+1} & =y_{n}+d x_{n}-b x_{n} y_{n} z_{n}, \\
z_{n+1} & =(1-m) x_{n}+m z_{n}, \\
u_{n+1} & =a+(1-b-d) u_{n}+b u_{n} v_{n} w_{n}+\gamma\left(u_{n}-x_{n}\right), \\
v_{n+1} & =v_{n}+d u_{n}-b u_{n} v_{n} w_{n}, \\
w_{n+1} & =(1-m) u_{n}+\frac{m b}{a} w_{n}^{2},
\end{aligned}
$$

where $\gamma$ is a constant that controls the coupling strength. We set the values of the parameters

$$
a=0.02, b=0.06, d=0.05, \text { and } m=0.05,
$$




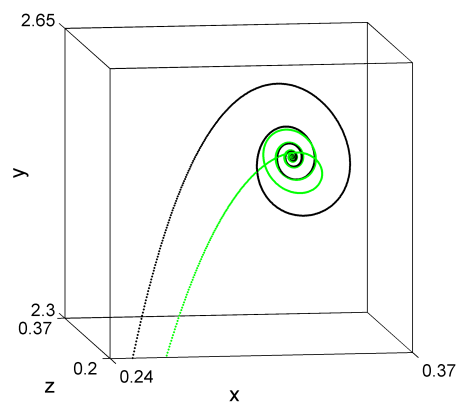

(a) $\gamma=0.005$

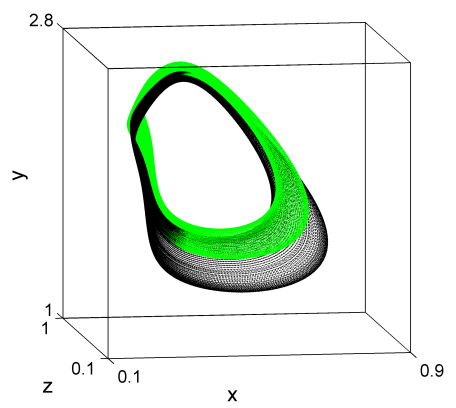

(d) $\gamma=0.0109$

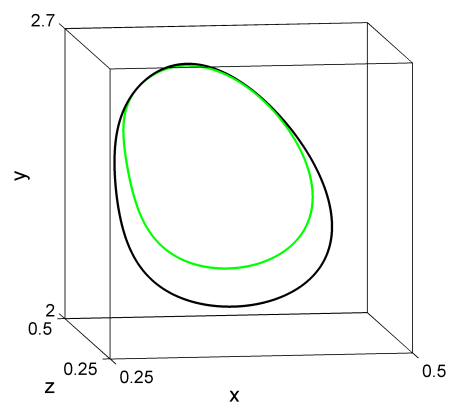

(b) $\gamma=0.008$

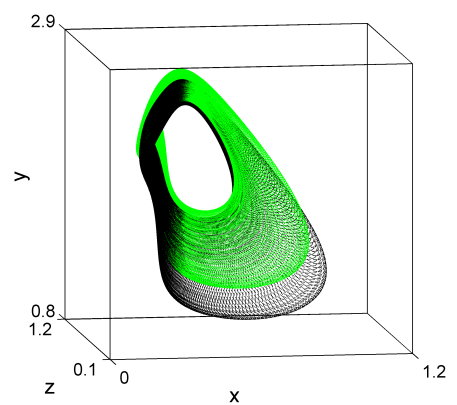

(e) $\gamma=0.012$

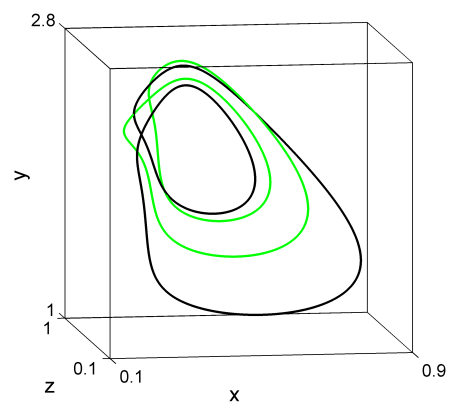

(c) $\gamma=0.01$

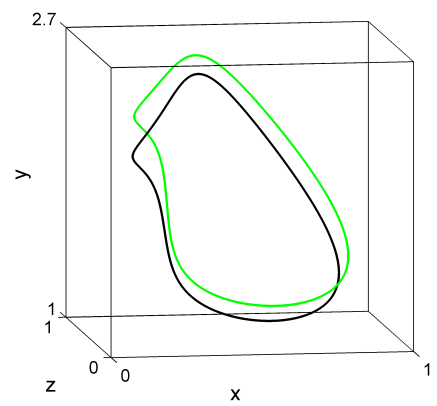

(f) $\gamma=0.015$

Fig. 7. CASE II in Sec. 5.2. The limiting behaviors of the orbits in $\mathbf{A}_{2}$ and $\mathbf{B}_{2}$ according to the coupling strength $\gamma$. The orbit of the system $\mathbf{A}_{2}$ is in black, and that of $\mathbf{B}_{2}$ in green in color.

and the initial points of $\mathbf{A}_{2}$ and $\mathbf{B}_{2}$ are both

$$
\left(x_{0}, y_{0}, z_{0}\right)=\left(u_{0}, v_{0}, w_{0}\right)=(0,-1200,0) .
$$

As for the initial points, unlike CASE I in Sec. 5.1, since the systems in the coupled system have distinct forms, it is not necessary to choose the different initial points for $\mathbf{A}_{2}$ and $\mathbf{B}_{2}$. When no coupling exists, i.e. $\gamma=0$, one can check that the systems $\mathbf{A}_{2}$ and $\mathbf{B}_{2}$ have both the same fixed point which is $(1 / 3,5 / 2,1 / 3)$.

As $\gamma$ increases from 0 to 0.02 , the following are observed, regarding the orbits of $\mathbf{A}_{2}$ and $\mathbf{B}_{2}$ in the coupled system with the initial conditions denoted in (5.23).

(1) When $0 \leq \gamma \leq 0.007$, the orbits converge both to the fixed point $(1 / 3,5 / 2,1 / 3)$. See Fig. 7 (a).

(2) When $0.008 \leq \gamma \leq 0.010$, both orbits approach but not converge to the fixed point. Each orbit rotates along a distinct simple closed curve around the fixed point. See Fig. 7 (b) and (c).

(3) When $0.010<\gamma \leq 0.013$, both orbits approach but not converge to the fixed point. They rotate around the fixed point with chaotic motion. See Fig. 7 (d) and $(\mathrm{e})$.

(4) When $0.014 \leq \gamma \leq 0.017$, both orbits rotate around the fixed point without converging to the fixed point, similarly to the case when $0.008 \leq \gamma \leq 0.010$. See 
Fig. 7 (f).

(5) When $0.018 \leq \gamma \leq 0.019$, both orbits approach the fixed point at the beginning, but eventually, they escape to infinity.

Note that even at the same coupling strength $\gamma$, two completely different behaviors may occur in the coupled system due to different initial conditions. For example, at $\gamma=0.0109$, the coupled system display two different rotating behaviors according to the initial values.

\subsection{CASE III}

We consider another coupled system $\left(\mathbf{A}_{3}, \mathbf{B}_{3}\right)$, which is composed of two systems in different forms and with different parameters, given by

$$
\mathbf{A}_{3}=\left\{\left(x_{k}, y_{k}, z_{k}\right)\right\}_{k=0}^{\infty} \text { and } \mathbf{B}_{3}=\left\{\left(u_{k}, v_{k}, w_{k}\right)\right\}_{k=0}^{\infty},
$$

where

$$
\begin{aligned}
& x_{n+1}=a_{1}+\left(1-b_{1}-d_{1}\right) x_{n}+b_{1} x_{n} y_{n} z_{n}+\gamma\left(x_{n}-u_{n}\right), \\
& y_{n+1}=y_{n}+d_{1} x_{n}-b_{1} x_{n} y_{n} z_{n}, \\
& z_{n+1}=\left(1-m_{1}\right) x_{n}+m_{1} z_{n}, \\
& u_{n+1}=a_{2}+\left(1-b_{2}-d_{2}\right) u_{n}+b_{2} u_{n} v_{n} w_{n}+\gamma\left(u_{n}-x_{n}\right), \\
& v_{n+1}=v_{n}+d_{2} u_{n}-b_{2} u_{n} v_{n} w_{n}, \\
& w_{n+1}=\left(\left(\sqrt{\frac{b_{2}}{a_{2}}}-m_{2}\right) u_{n}+m_{2} w_{n}\right)^{2} .
\end{aligned}
$$

We choose the following values of the parameters:

(1) $a_{1}=0.02, b_{1}=0.04, d_{1}=0.01$, and $m_{1}=0.05$.

(2) $a_{2}=0.04, b_{2}=0.08, d_{2}=0.02$, and $m_{2}=0.05$.

In addition, the initial conditions of $\mathbf{A}_{3}$ and $\mathbf{B}_{3}$ are both

$$
\left(x_{0}, y_{0}, z_{0}\right)=\left(u_{0}, v_{0}, w_{0}\right)=(0,-1900,0) .
$$

The systems $\mathbf{A}_{3}$ and $\mathbf{B}_{3}$ in the coupled system have the same fixed point $(0.5,0.5,0.5)$ when $\gamma=0$. Similarly to the examples in the previous cases, depending on the coupling strength, the orbits of $\mathbf{A}_{3}$ and $\mathbf{B}_{3}$ may converge both to the fixed point, or converge to the distinct points, or escape to infinity. In the case that the systems in the coupled system converge, the orbit of $\mathbf{B}_{3}$ approaches the point more quickly than that of $\mathbf{A}_{3}$, because the parameters $a_{1}$ and $a_{2}$ are proportional to the rate of convergence and $a_{1}<a_{2}$. See Fig. 8 (a). It is observed that when $0 \leq \gamma \leq 0.02$, both $\mathbf{A}_{3}$ and $\mathbf{B}_{3}$ converges to the fixed point, and when $\gamma \geq 0.03$ they escape to infinity. Figure 8 (b) illustrates that the orbit of $\mathbf{B}_{3}$ approaches the fixed point more quickly than that of $\mathbf{A}_{3}$ at the beginning, but in a neighborhood of the fixed point the orbit of $\mathbf{B}_{3}$ becomes slower and that of $\mathbf{A}_{3}$ becomes faster, and finally, both orbits converge to the fixed point simultaneously. 


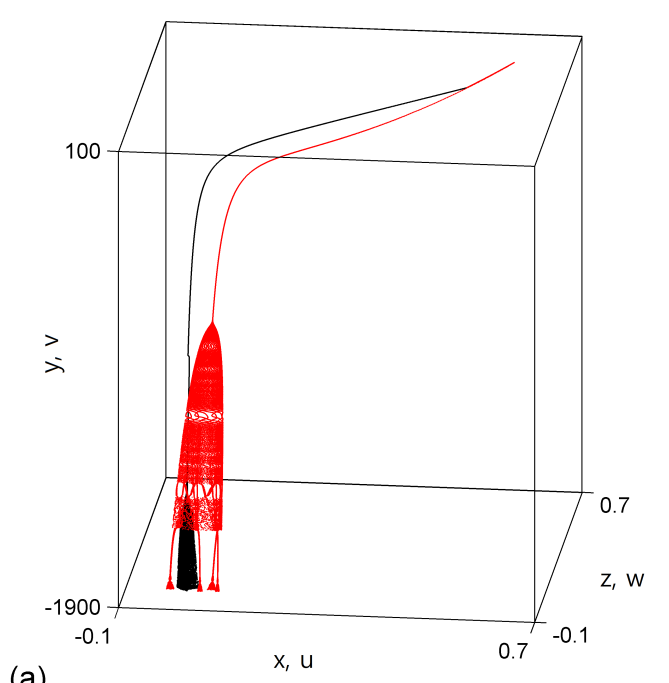

(a)

Fig. 8. CASE III in Sec. 5.3. The coupling strength is given by $\gamma=0.01$. Two orbits of $\mathbf{A}_{3}$ and $\mathbf{B}_{3}$ are in black and in red (in color), respectively. (a) Both orbits converge to the fixed point $(1 / 2,1 / 2,1 / 2)$. (b) Time series of the sequences $\left\{x_{n}\right\}$ and $\left\{u_{n}\right\}$ of the two orbits in $\mathbf{A}_{3}$ and $\mathbf{B}_{3}$ from $n=1$ to $n=130000$.

\section{Conclusions}

From a mathematical point of view, the present model shows the process of convergence to the fixed point, depending on the initial conditions. The model shows the complexity of orbits, whose complexity stems from the universality of direction fields (discretized version of vector fields). Furthermore, the complexity contains varieties of bifurcations.

The present study provides an computational model for a mirror neuron or a mirror-neuron system, which has recently been highlighted in neuroscience. However, according to the present results, it is necessary to experience complex bifurcations via the Hopf bifurcation for identifying other individuals' movements with own movements. Furthermore, the system has to experience a long time transient motions till the reach to the equilibrium state which represents a completely assimilated state. If a rapid rate of convergence to such a final state is demanded, the selection of the initial conditions is prerequisite. Another algorithm of selecting demanded initial conditions is necessary. In order to perform this task, a further function such as a cost function should be introduced, thereby the problem should be treated as a functional analysis via a variational principle, by introducing, say, genetic algorithms. This problem is for the future studies.

On the other hand, the proposed computational model for mirror-neuron systems may have a neuronal basis. Recently, functional relationship between human mirror-neuron systems and $\mu$-wave of $8-13 \mathrm{~Hz}$ oscillations has been investigated [8], in particular, as elucidating the characteristics of autism. In normal subjects, $\mu$-wave 
in premotor area disappears when mirror-neuron systems are working, whereas it remains in autistic patients [1]. Although the dynamical mechanism of this type of oscillations is still unclear, the oscillations can be yielded by Hopf bifurcations and more complex behaviors such as chaotic itinerancy can appear in its coupled systems [12]. Therefore, the proposed model can be justified by the evidence of the presence of Hopf bifurcations in EEG data.

\section{Acknowledgments}

One of the authors (I. Tsuda) is partially supported by a Grant-in-Aid for Scientific Research on Innovative Areas "The study on the neural dynamics for understanding communication in terms of complex hetero systems (No.4103)" (2112002) of The Ministry of Education, Culture, Sports, Science, and Technology, Japan. The other author (H. Kang) is partially supported for this work by the Soongsil University Research Fund of 2012.

\section{References}

[1] Cherman L, Hubbard E, McCleery J, Alschuler E, Pineda F, Ramachandran V, EEG evidence for mirror neuron dysfunction in autism spectrum disorders, Cognitive Brain Res 24:190-198, 2005.

[2] Guckenheimer J, Persistent properties of bifurcations, Phys D 7:105-110, 1983.

[3] Guckenheimer J, Holmes P, Nonlinear Oscillations, Dynamical systems, and Bifurcations of Vector Fields, Springer-Verlag, 1983.

[4] Kang H, Dynamics of local map of a discrete brusselator model: Eventually trapping regions and strange attractors, Discrete Contin Dyn Syst 20:939-959, 2008.

[5] Kang H, Tsuda I, On embedded bifurcation structure in some discretized vector fields, CHAOS 19:033132, 2009.

[6] Kataoka N, Kaneko K, Functional dynamics I: Articulation process, Phys D 138:225$250,2000$.

[7] Marsden J, McCracken M, The Hopf Bifurcation and its Applications, Springer, 1976.

[8] Mizuhara H, Inui T, Advances in Cognitive Neurodynamics (II): Is mu rhythm an index of the human mirror neuron system? A study of simultaneous fMRI and EEG, Springer, 2009.

[9] Rizzolatti G, Craighero L, The Mirror-Neuron system, Annu Rev Neurosci 27:169-192, 2004.

[10] Rizzolatti G, Sinigaglia L, Mirrors in the Brain. How We Share our Actions and Emotions, Oxford University Press, 2008.

[11] Thompson J, Stewart H, Nonlinear Dynamics and Chaos, Wiley, New York, 1986.

[12] Tsuda I, Fujii H, Tadokoro S, Yasuoka T, Yamaguti Y, Chaotic itinerancy as a mechanism of irregular changes between synchronization and desynchronization in a neural network, J Interg Neurosci, 3:159-182, 2004. 